\title{
Implementing a robotic liver resection program does not always require prior laparoscopic experience
}

\author{
Emanuele Balzano ${ }^{1}$ - Lorenzo Bernardi ${ }^{1}$ [D . Giovanni Tincani ${ }^{1}$. Davide Ghinolfi ${ }^{1} \cdot$ Fabio Melandro ${ }^{1}$. \\ Jessica Bronzoni ${ }^{1}$. Sonia Meli ${ }^{1}$. Giuseppe Arenga ${ }^{1}$. Giandomenico Biancofiore ${ }^{2,4}$ - Laura Crocetti ${ }^{3,4}$. \\ Paolo De Simone ${ }^{1,4}$
}

Received: 26 January 2021 / Accepted: 16 July 2021 / Published online: 4 October 2021

(C) The Author(s) 2021

\begin{abstract}
Background Preliminary experience in laparoscopic liver surgery is usually suggested prior to implementation of a robotic liver resection program.

Methods This was a retrospective cohort analysis of patients undergoing robotic (RLR) versus laparoscopic liver resection (LLR) for hepatocellular carcinoma at a center with concomitant initiation of robotic and laparoscopic programs

Results A total of 92 consecutive patients operated on between May 2014 and February 2019 were included: 40 RLR versus 52 LLR. Median age (69 vs. $67 ; p=0.74)$, male sex (62.5\% vs. 59.6\%; $p=0.96)$, incidence of chronic liver disease $(97.5 \%$ vs.98.1\%; $p=0.85$ ), median model for end-stage liver disease (MELD) score ( 8 vs. $9 ; p=0.92)$, and median largest nodule size ( 22 vs. $24 \mathrm{~mm}$ ) were similar between RLR and LLR. In the LLR group, there was a numerically higher incidence of nodules located in segment 4 ( $20.0 \%$ vs. $16.6 \% ; p=0.79)$; a numerically higher use of Pringle's maneuver $(32.7 \%$ vs. $20 \%$; $p=0.23$ ), and a shorter duration of surgery (median of 165.5 vs. $217.5 \mathrm{~min} ; p=0.04$ ). Incidence of complications (25\% vs. $32.7 \% ; p=0.49)$, blood transfusions $(2.5 \%$ vs.9.6\%; $p=0.21)$, and median length of stay $(6$ vs. $5 ; p=0.54)$ were similar between RLR and LLR. The overall (OS) and recurrence-free (RFS) survival rates at 1 and 5 years were 100 and 79 and 95 and $26 \%$ for RLR versus 96.2 and 76.9 and 84.6 and $26.9 \%$ for LLR (log-rank $p=0.65$ for OS and 0.72 for RFS).

Conclusions Based on our results, concurrent implementation of a robotic and laparoscopic liver resection program appears feasible and safe, and is associated with similar oncologic long-term outcomes.
\end{abstract}

Keywords Hepatocellular carcinoma $\cdot$ Liver resection $\cdot$ Robot $\cdot$ Learning curve $\cdot$ Outcomes

Liver resection is the backbone of the management of hepatocellular carcinoma (HCC), with the potential for radicality in early-stage tumors and well-selected candidates [1]. While open liver resections (OLR) have largely expanded in

Paolo De Simone

paolo.desimone@unipi.it

Emanuele Balzano

e.balzano@ao-pisa.toscana.it

1 Hepatobiliary Surgery and Liver Transplantation, University of Pisa Medical School Hospital, Pisa, Italy

2 Intensive Care Unit, University of Pisa Medical School Hospital, Pisa, Italy

3 Interventional Radiology, University of Pisa Medical School Hospital, Pisa, Italy

4 Department of Surgical, Medical, Biochemical Pathology and Intensive Care, University of Pisa, Pisa, Italy the Nineties, minimally invasive liver surgery (MILS)—such as robotic (RLR) and laparoscopic liver resections (LLR) have been refined only more recently [2,3]. The advantages of MILS have largely been described in the literature, and MILS has gained wider consent among health care professionals [4, 5]. Compared to open surgery, RLR and LLR are associated with less intraoperative blood losses, lower rates of complications, and shorter length of postoperative stay due to faster patient recovery [6, 7]. In particular, MILS is associated with a reduced risk of postoperative ascites and liver failure in patients with cirrhosis [8], while providing comparable oncological outcomes to OLR [5, 7, 9].

Superiority of either MILS technique has not yet been confirmed [10,11]. Similar safety and oncologic outcomes have been reported $[12,13]$, but the robotic approach allows to overcome some technical limitations of laparoscopy, such as access to posterior and superior liver segments, which 
may be technically demanding in LLR [14-16]. Finally, the learning curve seems to be shorter for RLR when compared to laparoscopy $[17,18]$.

Usually, robot-assisted surgery programs are implemented only after experience in laparoscopy has been achieved [19]. This was not the case at our department, where a robotic liver resection program was implemented concomitantly with laparoscopic liver surgery. The aim of the current paper is to illustrate the results of our initial experience with RLR versus LLR, discuss the feasibility of a robotic liver surgery program without prior experience in major laparoscopic liver surgery, and present the safety and oncological efficacy of both the procedures for the treatment of HCC.

\section{Materials and methods}

\section{Study design and setting}

This was a retrospective cohort analysis of consecutive patients undergoing RLR versus LLR for the treatment of $\mathrm{HCC}$ at the Hepatobiliary surgery and Liver transplantation Unit of the University of Pisa Medical School Hospital between May 2014 and February 2019. Data were derived from a prospectively collected database and retrospectively reviewed with focus on the characteristics of the population, tumors, and surgical procedures. The primary endpoint of our analysis was to assess the feasibility, safety, and oncological efficacy of both RLR and LLR as per conversion rates, incidence of complications, and overall (OS) and recurrence-free survival (RFS) rates. Type and severity of complications were classified according to Dindo-Clavien [20]. Blood transfusions included the intraoperative and postoperative period. We also assessed the distribution of complications per surgeon and population quartiles to account for the individual and institutional learning curve.

\section{Indications to surgery}

The preoperative diagnosis of HCC was according to the European Association for the Study of the Liver (EASL) guidelines [21, 22]. In the current experience, RLR and LLR were both reserved to patients with compensated chronic liver disease (i.e., Child-Pugh A or B cirrhosis), tumors within Milan criteria and/or lesions not amenable to local ablative treatment (as per subcapsular site or proximity with major vascular and biliary structures). In contrast with the most recent recommendations, the presence of portal hypertension (i.e., either splenomegaly associated with platelet count $<100,000 / \mathrm{mm}^{3}$ or esophageal varices) was not considered an exclusion criterion to either procedure [21]. Chronic treatment with antiplatelet drugs was not interrupted before surgery.

\section{Implementation of the surgical program}

Prior to initiation of the current programs, our senior surgical staff (EB, GT, DG, FB, GC, GA, PDS) had achieved experience in minor laparoscopic procedures (cholecystectomy; appendectomy), non-resective liver surgeries (i.e., cyst fenestration), and colic resection. Implementation of RLR and LLR was preceded by participation of all surgeons to accredited international programs, and the first 10 robotic procedures were tutored by a senior surgeon from an external institution. No external mentoring was performed for LLR.

\section{Statistical analysis}

According to their level of measurement and distribution, continuous variables were expressed as means and standard deviations (SD) or medians and ranges, while categorical variables are described as frequencies. Data were compared with the t-test for continuous values with normal distribution, the Mann-Whitney $U$ test for continuous values without normal distribution, and the Pearson's chi-square or Fisher's exact tests for categorical values. The level of significance was set at $5 \%$. Survival rates were obtained according to Kaplan-Meier at 1,2, and 5 years after surgery, and comparison between group was according to the log-rank. Survival was censored at death, latest follow-up, or at transplantation in the event of salvage liver transplantation (LT). The latter was indicated in the case of HCC recurrence or liver function decompensation. The study protocol was approved by the Institutional Review Board of the University Hospital of Pisa, Italy. The patients provided written informed consent to current analysis.

\section{Results}

A total of 92 patients were included: 40 underwent RLR as opposed to 52 treated with LLR. The demographic and clinical characteristics of interest of the 2 groups are illustrated in Table 1 . No statistically significant difference was observed for any of the clinical variables (Table 1). There was a numerically higher incidence of previous abdominal surgeries and antiplatelet use for RLR versus LLR, and a numerically higher frequency of nodules located in segment 4 for LLR patients (Table 1).

The total number of treated lesions was 98 (43 for RLR vs. 55 for LLR), and the median largest nodule diameter was 
Table 1 Demographic and clinical characteristics of the study populations
Table 2 Surgical data

\begin{tabular}{|c|c|c|c|}
\hline Variable & Robotic (\#40) & Laparoscopic (\#52) & $p$ \\
\hline Male, $n(\%)$ & $25(62.5)$ & $31(59.6)$ & 0.96 \\
\hline Age (years), median [range] & $69[46-83]$ & $67[34-86]$ & 0.74 \\
\hline BMI $\left(\mathrm{Kg} / \mathrm{m}^{2}\right)$, median [range] & $26.0[15.2-34.8]$ & $26.4[18.0-33.6]$ & 0.98 \\
\hline ASA score, median [range] & $3[1-4]$ & $3[1-4]$ & 0.99 \\
\hline Previous abdominal surgery, $n(\%)$ & $18(45.0)$ & $15(28.8)$ & 0.10 \\
\hline Antiplatelet treatment, $n(\%)$ & $9(22.5)$ & $6(11.5)$ & 0.15 \\
\hline Cirrhosis & $39(97.5)$ & $51(98.1)$ & 0.85 \\
\hline MELD score, median [range] ${ }^{\mathrm{a}}$ & $8[6-13]$ & $9[6-15]$ & 0.92 \\
\hline Total nodules, $n$ & 43 & 55 & \\
\hline Nodules per patient, median [range] & $1[2]$ & $1[3]$ & 0.98 \\
\hline Largest nodule diameter $(\mathrm{mm})$, median [range] & $22[12-65]$ & $24[11-58]$ & 0.97 \\
\hline \multicolumn{4}{|l|}{ Tumor nodule site, $n(\%)$} \\
\hline S2 & $2(4.7)$ & $4(7.4)$ & 0.69 \\
\hline S3 & $6(14.0)$ & $8(14.5)$ & 0.99 \\
\hline S4 & $7(16.3)$ & $11(20.0)$ & 0.79 \\
\hline S5 & $8(18.6)$ & $10(18.2)$ & 0.99 \\
\hline S6 & $9(20.9)$ & $14(25.4)$ & 0.63 \\
\hline S7 & $2(4.7)$ & $0(0)$ & 0.19 \\
\hline S8 & $9(20.9)$ & $8(14.5)$ & 0.43 \\
\hline
\end{tabular}

ASA American Society of Anesthesiology, MELD model for end-stage liver disease

${ }^{\text {a } M E L D}$ is provided for patients with cirrhosis

\begin{tabular}{llll}
\hline Procedure & Robotic (\#40) & Laparoscopic (\#52) & $p$ \\
\hline Wedge resection, $n(\%)$ & $36(90.0)$ & $44(84.6)$ & 0.44 \\
Segmentectomy, $n(\%)$ & $2(5.0)$ & $4(7.7)$ & 0.69 \\
Bisegmentectomy, $n(\%)$ & $2(5.0)$ & $4(7.7)$ & 0.69 \\
Pringle's maneuver, $n(\%)$ & $8(20.0)$ & $17(32.7)$ & 0.23 \\
Length (min), median [range] & $217.5[95.0-390.0]$ & $165.5[80.0-256.0]$ & 0.04 \\
Conversion, $n(\%)$ & $3(7.5)$ & $2(3.8)$ & 0.64 \\
Switch to ablation, $n(\%)$ & $2(5.0)$ & $0(0)$ & 0.18 \\
Patients transfused, $n(\%)$ & $1(2.5)$ & $5(9.6)$ & 0.21 \\
Blood loss $<200$ mL, $n(\%)$ & $39(97.5)$ & $47(90.4)$ & 0.22 \\
R0, $n(\%)$ & $36(90.0)$ & $46(88.5)$ & 0.99 \\
LOS (days), median [range] & $6[3-15]$ & $5[2-13]$ & 0.54 \\
Patients with complications ${ }^{\mathrm{a}}, n(\%)$ & $10(25.0)$ & $17(32.7)$ & 0.49 \\
Total complications ${ }^{\mathrm{a}}, n$ & 13 & 21 & \\
Grade 1 & 9 & 13 & 0.72 \\
Grade 2 & 1 & 3 & 0.99 \\
Grade 3 & 2 & 3 & 0.99 \\
Grade 4 & 1 & 2 & 0.99 \\
\hline
\end{tabular}

LOS length of stay

${ }^{\mathrm{a}}$ As per Dindo-Clavien

22 and $24 \mathrm{~mm}$ for RLR vs. LLR (Table 1). Surgical procedures are reported in Table 2 and consisted mainly of wedge resections (90\% for RLR vs. $84.6 \%$ for LLR, $p=0.44$ ). To note, use of Pringle's maneuver was numerically more frequent in LLR (32.7\% vs. 20.0; $p=0.23)$, and median duration of surgery was significantly longer for RLR versus LLR (217.5 vs. $165.5 \mathrm{~min} ; p=0.04$ ) (Table 2). Cholecystectomy was associated in $4(10.0 \%)$ RLR patients versus 9 (16.4\%) LLR, while one (2.5\%) RLR patient underwent 
simultaneous resection of a suspected tumor nodule located in the right kidney upper pole.

The conversion rate was numerically higher for RLR (7.5\% vs. $3.8 \% ; p=0.64)$, while in $2(5.0 \%)$ RLR patients with one nodule each the surgeon decided to switch the strategy from resection to radiofrequency ablation due to the presence of ascites. In the RLR group, reasons for conversion to open surgery were bleeding in $2(5.0 \%)$ cases (one from the liver parenchyma, and one from the combined kidney resection margin), and oxygen desaturation in one (2.5\%). Both conversions (3.8\%) in the LLR group were due to bleeding from the liver cut surface. Blood transfusions were numerically higher for LLR versus RLR $(9.6 \%$ vs. $2.5 \% ; p=0.21$ ).

In the RLR group, histology of 41 resected specimens was consistent with HCC in 39 (90.7\%), cholangiocarcinoma in one (2.3\%), and a fully necrotic nodule in a further one (2.3\%). In the LLR group, histology showed HCC in 54 (98.2\%) nodules, and mixed HCC-cholangiocarcinoma in one (1.8\%). R0 was achieved in $90.0 \%$ and $88.5 \%$ of RLR and LLR patients, respectively $(p=0.99)$ (Table 2). The median postoperative length of stay was 6 days for RLR as opposed to 5 for LLR $(p=0.54)$ (Table 2).

During the hospital stay, 10 (25.0\%) RLR patients presented a total of 13 complications versus 17 (32.7\%) LLR patients with 21 complications $(p=0.49)$. Grade $\geq$ III complications were observed in $7.5 \%$ RLR patients and consisted of one hematoma of the muscle rectus abdominis due to parietal bleeding at the level of the trocar port site and requiring radiology-guided embolization, ascites requiring percutaneous drainage, and surgical site active bleeding requiring open redo surgery (Table 2). Five (9.6\%) LLR patients presented grade $\geq$ III complications: two cases of ascites necessitating percutaneous drainage, two cases of abdominal collection requiring percutaneous drainage, and one surgical site active bleeding requiring open redo surgery (Table 2).

Median number of patients with complications per staff surgeon was 2 (range, $1-3$ ) in the RLR group versus 2.5 (range $1-3)$ in the LLR group $(p=0.78)$. Patient quartile breakdown analysis did not show any difference in the distribution of complications in any of the 2 groups. Namely, in the RLR group, the frequency of patients with complications was $3(7.5 \%), 2(5.0 \%), 3(7.5 \%)$, and $2(5.0 \%)$ in the first, second, third, and fourth quartiles $(p=0.98)$ versus $5(9.6 \%), 4(7.7 \%), 4(7.7 \%)$, and $4(7.7 \%)$ in the LLR group $(p=0.99)$.

In the RLR group, the median follow-up was 24 months (range, 8-57): 16 (40.0\%) patients presented HCC recurrence, one $(2.5 \%)$ such patient died due to recurrence, 2 (5.0\%) patients died for progression of liver disease, and one patient was lost to follow-up. Four (10.0\%) patients underwent salvage LT due to HCC recurrence and the median time from resection to LT was 8 months (range, 5-10).

In the LLR group, the median follow-up was 27.5 months (range, 9-55): 18 (34.6\%) patients presented HCC recurrence, 4 (7.7\%) such patients died to recurrence, $4(7.7 \%)$ died of progression of liver disease, and 2 (3.8\%) died of sepsis 15 and 18 months after surgery, respectively. Five (9.6\%) patients underwent salvage LT due to HCC recurrence and the median time from resection to $\mathrm{LT}$ was 11 months (range, 4-13).

The actuarial 1-, 2-, and 5-year OS and RFS were 100 and $79 \%$; 95 and $62 \%$; and 95 and $26.0 \%$ for RLR patients versus 96.2 and $76.9 \%$; 86.5 and $61.5 \%$; 84.6 and $26.9 \%$ for LLR (log-rank $p=0.65$ for OS and 0.72 for RFS). (Fig. 1).
Fig. 1 Kaplan-Meier estimates of overall (OS) and recurrencefree survival (RFS) in robotic (RLR) versus laparoscopic (LLR) liver resection for hepatocellular carcinoma

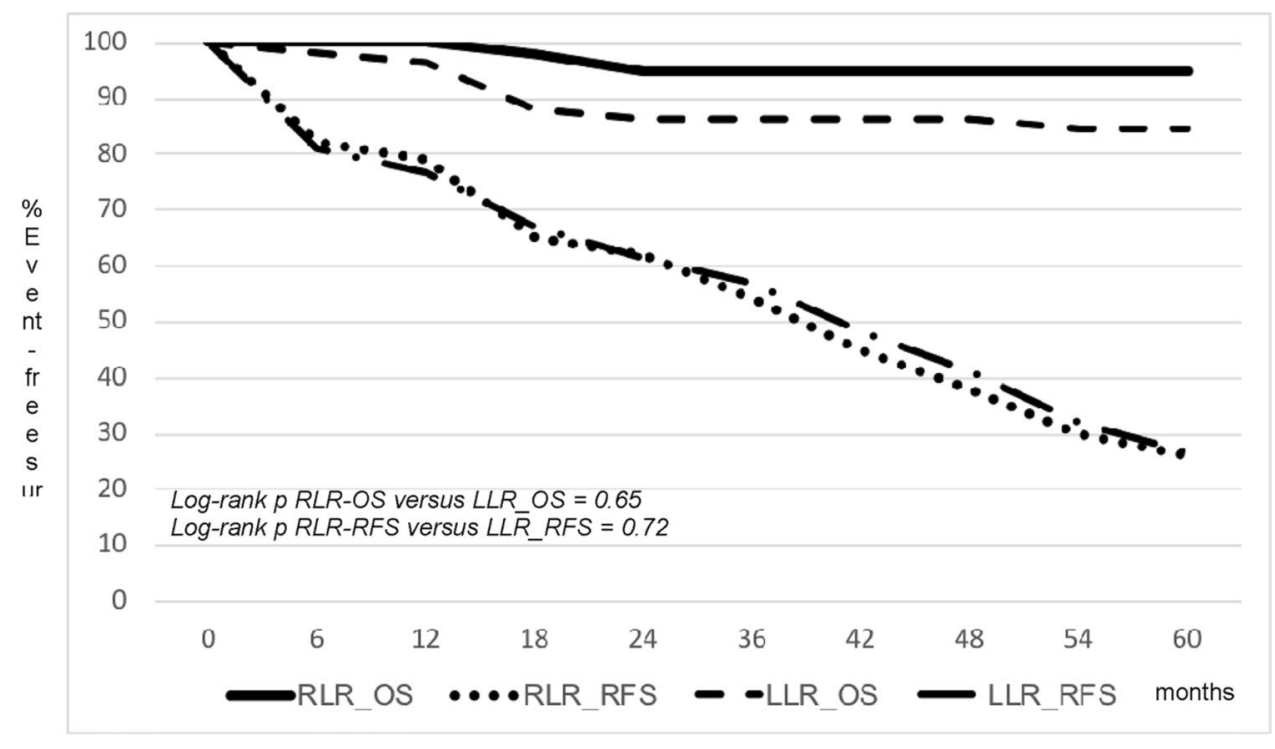




\section{Discussion}

With this retrospective cohort analysis, we aimed to evaluate the results of our initial experience with MILS for HCC, whether it be in the form of RLR or LLR. Unlike the majority of robot-assisted surgery programs, which are usually implemented once solid experience with LLR has been achieved, our MILS program consisted of concomitant implementation of either technique. Based on current results, preliminary experience with LLR might not be a necessary pre-requisite to robot-assisted liver surgery, although some requirements are to be complied with, such as extensive experience in open liver surgery and preliminary training in both the MILS techniques.

From a technical standpoint, however, some differences between RLR and LLR have to be underscored. The 7-degree motion of robotic arms provides excellent dexterity, and allows easier access to posterior and superior liver segments (S7, S8, S4b) when compared to LLR [16]. This might account for the fact that, despite similar prevalence of cirrhosis and concomitant medication with antiplatelet drugs in both groups, hilar clamping was necessary only in $20 \%$ of cases of RLR patients versus $32.7 \%$ for LLR. In the remainder of RLR patients, it was possible to perform clamp-free parenchymal dissection with very low transfusion rate $(2.5 \%)$ and non-significant blood losses $(<200 \mathrm{~mL})$ in all cases but one. The increased level of dexterity of robot-assisted surgery might also explain why previous open abdominal surgery-which was reported in 45\% (18/40) of RLR patients-did not seem to affect its feasibility or safety rates of RLR, nor its oncological efficacy. In such cases, careful dissection of adherences could be carried out and inadvertent visceral injuries were avoided. On the opposite, it is important to note that RLR was a longer procedure than LLR, since duration of surgery is influenced by preliminary (docking) phases and device repositioning during the varied phases of surgical procedures. No major impact was observed on length of hospital stay, which was a median of 6 and 5 days, respectively, for RLR and LLR. This might reflect the initial learning curve phase of our institution, since no difference was observed between the two cohorts.

Even if the present study elucidated the absence of differences in terms of feasibility or safety of RLR versus LLR for the treatment of HCC, it did not allow to clarify the duration of each individual surgeon learning curve with either technique. The learning curve is a rather complex process which is influenced by surgeon, patient, and institution-derived correlates and it deserves properly powered studies and investigations. Our experience was biased by the concomitant practice of open liver surgery and transplantation, as well as by the level of efficiency achieved by the anesthesiology and nursing teams. All of these variables, alongside a detailed case-mix analysis, should be included in future trials to unveil the intricacies underlying the learning curve effect.

Despite the technical differences between RLR and LLR, their oncologic efficacy is comparable. In both populations, we achieved similar overall and recurrence-free survival rates, and the majority of recurrences occurred 2 years after surgery in either group. These data are consistent with the high prevalence of cirrhosis among our patients. However, OS plateaued 2 years after surgery, thanks to implementation of treatment strategies for HCC recurrences (data not shown), including radiologic treatments, oral drugs, and ultimately LT [1]. Of interest, 4 (10.0\%) RLR and 5 (9.6\%) LLR patients underwent salvage LT at a median interval of 8 and 11 months after primary surgery, respectively. To this regard, Guerrini et al. have reported that salvage LT is a viable treatment option for local recurrences, and that previous RLR does not seem to impair the long-term outcome of transplantable cirrhotic patients affected with recurrent HCC [23]. However, only a third of these patients can benefit from salvage transplantation, suggesting that patient selection for a "resection-first" policy together with prompt recurrence detection are both key to improving the results of liver resection followed by salvage LT [24]. Based on the low rate of complications and the comparable oncological efficacy of MILS versus open surgery, RLR and LLR deserve further investigation as bridging modalities prior to LT in order to protect patients from the risk of wait list drop out or as a resection-first strategy followed by salvage LT in the event of local recurrence [25, 26].

In conclusion, our experience confirms that RLR and LLR can be implemented concurrently for the treatment of HCC in compensated cirrhotic patients and in centers with extensive liver surgery practice. Both techniques provide similar efficacy, with the potential to overcome the burden of open surgery and preserve eventual eligibility to transplantation. Based on their advantages, MILS deserves to be investigated as the elective pre-liver transplant bridging modality for those patients with resectable HCC and anticipated longer wait listing times.

Funding Open access funding provided by Università di Pisa within the CRUI-CARE Agreement.

\section{Declarations}

Disclosures Drs. Emanuele Balzano, Lorenzo Bernardi, Giovanni Tincani, Davide Ghinolfi, Fabio Melandro, Jessica Bronzoni, Sonia Meli, Giuseppe Arenga, Giandomenico Biancofiore, Laura Crocetti, 
and Paolo De Simone have no conflict of interest or financial ties to disclose.

Open Access This article is licensed under a Creative Commons Attribution 4.0 International License, which permits use, sharing, adaptation, distribution and reproduction in any medium or format, as long as you give appropriate credit to the original author(s) and the source, provide a link to the Creative Commons licence, and indicate if changes were made. The images or other third party material in this article are included in the article's Creative Commons licence, unless indicated otherwise in a credit line to the material. If material is not included in the article's Creative Commons licence and your intended use is not permitted by statutory regulation or exceeds the permitted use, you will need to obtain permission directly from the copyright holder. To view a copy of this licence, visit http://creativecommons.org/licenses/by/4.0/.

\section{References}

1. Forner A, Reig M, Bruix J (2018) Hepatocellular carcinoma. Lancet. https://doi.org/10.1016/S0140-6736(18)30010-2

2. Giulianotti PC (2003) Robotics in general surgery: personal experience in a large community hospital. Arch Surg 138(7):777

3. Giulianotti PC, Coratti A, Sbrana F, Addeo P, Bianco FM, Buchs NC et al (2011) Robotic liver surgery: results for 70 resections. Surgery 149(1):29-39

4. Han DH, Choi SH, Park EJ, Kang DR, Choi GH, Choi JS (2016) Surgical outcomes after laparoscopic or robotic liver resection in hepatocellular carcinoma: a propensity-score matched analysis with conventional open liver resection: minimally invasive liver resection for hepatocellular carcinoma. Int J Med Robot 12(4):735-742

5. Liu R, Wakabayashi G, Kim H-J, Choi G-H, Yiengpruksawan A, Fong $\mathrm{Y}$ et al (2019) International consensus statement on robotic hepatectomy surgery in 2018. World J Gastroenterol 25(12):1432-1444

6. Giulianotti PC, Bianco FM, Daskalaki D, Gonzalez-Ciccarelli LF, Kim J, Benedetti E (2016) Robotic liver surgery: technical aspects and review of the literature. Hepatobiliary Surg Nutr 5(4):311-321

7. Chen P-D, Wu C-Y, Hu R-H, Chou W-H, Lai H-S, Liang J-T et al (2017) Robotic versus open hepatectomy for hepatocellular carcinoma: a matched comparison. Ann Surg Oncol 24(4):1021-1028

8. Prodeau M, Drumez E, Duhamel A, Vibert E, Farges O, Lassailly $\mathrm{G}$ et al (2019) An ordinal model to predict the risk of symptomatic liver failure in patients with cirrhosis undergoing hepatectomy. $\mathbf{J}$ Hepatol 71(5):920-929

9. Yamashita S, Conrad C (2017) Robotic hepatectomy: a new paradigm in the management of hepatocellular carcinoma? Ann Surg Oncol 24(4):866-867

10. Memeo R, de Angelis N, de Blasi V, Cherkaoui Z, Brunetti O, Longo $\mathrm{V}$ et al (2016) Innovative surgical approaches for hepatocellular carcinoma. World J Hepatol 8(13):591

11. Lim C, Salloum C, Tudisco A, Ricci C, Osseis M, Napoli N et al (2019) Short- and long-term outcomes after robotic and laparoscopic liver resection for malignancies: a propensity scorematched study. World J Surg 43(6):1594-1603
12. Montalti R (2015) Outcomes of robotic vs laparoscopic hepatectomy: a systematic review and meta-analysis. World J Gastroenterol 21(27):8441

13. Guan R, Chen Y, Yang K, Ma D, Gong X, Shen B et al (2019) Clinical efficacy of robot-assisted versus laparoscopic liver resection: a meta analysis. Asian J Surg 42(1):19-31

14. Morise Z (2016) Laparoscopic liver resection for posterosuperior tumors using caudal approach and postural changes: A new technical approach. World J Gastroenterol 22(47):10267

15. Cheung TT, Han H-S, She WH, Chen K-H, Chow PKH, Yoong BK et al (2018) The Asia Pacific consensus statement on laparoscopic liver resection for hepatocellular carcinoma: a report from the 7th Asia-Pacific primary liver cancer expert meeting held in Hong Kong. Liver Cancer 7(1):28-39

16. Boggi U, Caniglia F, Vistoli F, Costa F, Pieroni E, Perrone VG (2015) Laparoscopic robot-assisted resection of tumors located in posterosuperior liver segments. Updat Surg 67(2):177-183

17. Efanov M, Alikhanov R, Tsvirkun V, Kazakov I, Melekhina O, Kim P et al (2017) Comparative analysis of learning curve in complex robot-assisted and laparoscopic liver resection. HPB 19(9):818-824

18. Magistri P, Guerrini GP, Ballarin R, Assirati G, Tarantino G, Di Benedetto $F$ (2019) Improving outcomes defending patient safety: the learning journey in robotic liver resections. BioMed Res Int 2019:1-8

19. Lai ECH, Tang CN, Yang GPC, Li MKW (2011) Multimodality laparoscopic liver resection for hepatic malignancy-from conventional total laparoscopic approach to robot-assisted laparoscopic approach. Int J Surg 9(4):324-328

20. Dindo D, Demartines N, Clavien P-A (2004) Classification of surgical complications: a new proposal with evaluation in a cohort of 6336 patients and results of a survey. Ann Surg 240(2):205-213

21. EASL (2018) EASL clinical practice guidelines: management of hepatocellular carcinoma. J Hepatol 69:182-236

22. Marrero JA, Kulik LM, Sirlin CB, Zhu AX, Finn RS, Abecassis MM et al (2018) Diagnosis, staging, and management of hepatocellular carcinoma: 2018 practice guidance by the American association for the study of liver diseases. Hepatology 68(2):723-750

23. Guerrini GP, Gerunda GE, Montalti R, Ballarin R, Cautero N, De Ruvo N et al (2014) Results of salvage liver transplantation. Liver Int 34(6):e96-e104

24. Bhangui P, Allard MA, Vibert E, Cherqui D, Pelletier G, Cunha AS et al (2016) Salvage versus primary liver transplantation for early hepatocellular carcinoma: do both strategies yield similar outcomes? Ann Surg 264(1):155-163

25. Magistri P, Tarantino G, Ballarin R, Coratti A, Di Benedetto F (2017) Robotic liver surgery is the optimal approach as bridge to transplantation. World J Hepatol 9(4):224

26. Magistri P, Olivieri T, Assirati G, Guerrini GP, Ballarin R, Tarantino $G$ et al (2019) Robotic liver resection expands the opportunities of bridging before liver transplantation. Liver Transpl 25(7):1110-1112

Publisher's Note Springer Nature remains neutral with regard to jurisdictional claims in published maps and institutional affiliations. 